\title{
Process and Practice
}

Jeanne E. Bishop

Westlake Schools Planetarium, 24525 Hilliard Road, Westlake, Ohio 44145, U.S.A.

I would like to discuss strategies for participation within planetarium programs. The planetarium is not only a theater in which passive audiences can hear lectures and see demonstrations. It is also a classroom in which people of all ages can benefit from involvement. Participatory programs have been advocated in the US since the early 1970's, and the idea has grown and spread.

One participatory method involves drawing. For example, an elementary school group can draw a planet group for two or three different times. The psychological value of learning by drawing is well known. And drawing positions of stars and planets with appropriate labels produces take-home sky charts. Families can and do use them to learn astronomy and enjoy the sky together.

Drawing can be combined effectively with hypothesis-testing (predicting what one thinks will happen). In a lesson about the daily path of the sun at the beginnings of equinoxes and solstices, students can draw sunrise, noon, and sunset positions for each date, always being asked to predict from their previous observations (and drawings) where the sun will be next. A lesson on positions, shapes, and orientations of lunar phases is very effective with drawing and predictions.

With interactive questioning and hypothesis testing, the instructor quickly learns helpful learning characteristics about the audience: misconceptions, level of abstractive ability, and problems with visual perception ability. (An example of a perceptual problem is a student saying that the moon "looks like the capital letter ' $\mathrm{D}$ "' when, in fact, it is the last quarter moon presented in the sky.)

Use of model materials is also useful in the planetarium. With flashlights and small spheres, pairs of students can recreate the reasons for the phases of the moon at each step of change of phase in the planetarium sky. The topics of seasons, planet motions, and ideas of celestial sphere are also enhanced by student-manipulated models. Where it is impractical for students to all have model materials, the instructor can move or have students move one demonstration model. Dynamic human models can also be used (See my paper, "Dynamic Human Models," elsewhere in these proceedings.)

Other types of participation activities include role-playing and singing. Roleplaying means that students assume the names or designations of particular characters from history or made-up roles in plays or contrived situations. Examples are navigators in historical voyages, Galileo discovering with his small telescope, and a government body discussing pros and cons (a cost-benefit analysis) of a costly space project.

Very young children love to sing. They remember ideas well when they are attached to music. Instructor-written words that match well-known tunes can be learned quickly - songs about directions, star patterns with mythology, changing moon phases, and day-and-night are both fun and educational. Commercially- 
produced records of children's songs about the sky are also available.

There probably are as many participatory styles for planetarium teaching as there are styles in a normal classroom, and then a few more. The latest addition to participation techniques - initiated by one of our panelists, Terence Murtagh - is interactive decisions on topics to explore in a planetarium program. Measuring, using data to calculate answers to mathematical problems, creative writing under stars with a musical or natural-sounds background, and fantasy journeys in which audience members close their eyes and use imaginations to extend what the planetarium can demonstrate - perhaps a mind trip into a black hole, complete with references to relativity, aching feelings as one is stretched, and reflections on the predicament - all can be successfully used in the planetarium classroom and theater.

\section{Project STARWALK}

Bob Riddle

Lakeview Museum Planetarium, 1125 W. Lake Ave., Peoria, Illinois 81614, U.S.A.

Project STARWALK is an Earth/space science program, developed for elementary students in grades 3 and 5 or 4 and 6 . The program is designed around the teaching of some basic earth science concepts (Earth's rotation, revolution, and axial tilt), and their consequences. Classroom lessons are designed to both prepare and follow-up student visits to a planetarium facility. The planetarium is used as an instrument to display models to help the students understand the concepts. There are three visits to the planetarium (fall, winter, and spring).

The role of the teacher is to prepare the students for a laboratory experience at the planetarium. This is done by way of the materials provided, and whatever other strategies the teacher deems necessary. There are a variety of activities designed for both pre- and post-reinforcement of the concepts.

An integral part of Project STARWALK is the teacher preparation. This is done by way of a two-day inservice workshop, one day for each grade involved, and is conducted at a planetarium facility. Administrators and other grade-level teachers are welcome to attend as well. The participants are introduced to the teacher materials and the concepts and objectives of the program. They will be involved with the same activities as their students. Emphasis will be placed on understanding basic astronomy and earth-science concepts as they relate to the objectives of the program. The use of models, visual aids, and computers as aids in enhancing student comprehension are also part of the teacher workshop.

Project STARWALK is one of many programs within a nation-wide network known as the National Diffusion Network. It is funded by a dissemination grant from the US Department of Education. Within the National Diffusion Network are state contact people who help in coordinating efforts between projects like STARWALK 